\title{
Combinatorial vs. Algebraic Characterizations of Completely Pseudo-Regular Codes
}

\author{
M. Cámara, J. Fàbrega, M.A. Fiol, and E. Garriga * \\ Departament de Matemàtica Aplicada IV \\ Universitat Politècnica de Catalunya \\ Jordi Girona 1-3, Mòdul C3, Campus Nord \\ 08034 Barcelona, Catalonia (Spain) \\ \{mcamara, jfabrega,fiol, egarriga\}@ma4.upc.edu
}

Submitted: Nov 16, 2009; Accepted: Feb 25, 2010; Published: Mar 8, 2010

Mathematics Subject Classification: 05C50, 05E30

\begin{abstract}
Given a simple connected graph $\Gamma$ and a subset of its vertices $C$, the pseudodistance-regularity around $C$ generalizes, for not necessarily regular graphs, the notion of completely regular code. We then say that $C$ is a completely pseudoregular code. Up to now, most of the characterizations of pseudo-distance-regularity has been derived from a combinatorial definition. In this paper we propose an algebraic (Terwilliger-like) approach to this notion, showing its equivalence with the combinatorial one. This allows us to give new proofs of known results, and also to obtain new characterizations which do not depend on the so-called $C$-spectrum of $\Gamma$, but only on the positive eigenvector of its adjacency matrix. Along the way, we also obtain some new results relating the local spectra of a vertex set and its antipodal. As a consequence of our study, we obtain a new characterization of a completely regular code $C$, in terms of the number of walks in $\Gamma$ with an endvertex in $C$.
\end{abstract}

\section{Preliminaries}

Pseudo-distance-regularity is a natural generalization of distance-regularity which extends this notion to not necessarily regular graphs. The key point of this generalization relays

\footnotetext{
*Research supported by the "Ministerio de Ciencia e Innovación" (Spain) with the European Regional Development Fund under projects MTM2008-06620-C03-01 and by the Catalan Research Council under project 2005SGR00256.
} 
on defining an adequate weight for each vertex in such a way that we obtain a "regularized" graph. Since its introduction in [7], the study of pseudo-distance-regularity produced several interesting results, specially in the area of quasi-spectral characterizations of distance-regularity $[4,7]$ and completely regular codes $[5,6]$. This study was based on the combinatorial definition of pseudo-distance-regularity around a vertex, which comes up naturally from the notion of distance-regularity around a vertex. Among the variety of techniques used in these works, two concepts stand out: the local spectrum (of a single vertex or a subset of vertices) and certain families of orthogonal polynomials.

Our work in this paper is motivated by the connection existing between pseudodistance-regularity and the study developed by Terwilliger [11] in the context of association schemes. In his work, he introduced the subconstituent algebra (also known as Terwilliger algebra) with respect to a vertex of a graph and defined the notion of thin module in this algebra. As commented by the third and fourth authors in $[3,5]$, the concept of pseudo-distance-regularity around a vertex $i$ is equivalent to the thin character of the minimum module containing its characteristic vector $\boldsymbol{e}_{i}$. The aim of this paper is to extend this parallelism from a single vertex to a set of vertices.

The plan of the paper is as follows. In the rest of this section we first give some notation on graphs and their spectra. In Section 2 we introduce the local spectrum of a vertex set, discussing some of its properties. Special attention is paid to the relation between the local spectra of two antipodal subsets of vertices. Section 3 is devoted to explain the concept of pseudo-distance-regularity around a vertex set, in combinatorial sense, and to review some of its known quasi-spectral characterizations. In the case of regular graphs, this concept coincides with that of a completely regular code. According to this fact, we say that a set of vertices satisfying this property is a completely pseudo-regular code. Our main results are in Section 4, where we extend the (algebraic) definition of Terwilliger to a set of vertices in any graph, and prove its equivalence with the combinatorial approach. This allows us to give new proofs of known results, and also to obtain new characterizations which do not depend on the so-called $C$-local spectrum, but only on the positive eigenvector of the adjacency matrix. As a consequence, we obtain a new characterization of a completely regular code $C$, in terms of the number of walks having an endvertex in $C$.

Throughout this paper $\Gamma=(V, E)$ stands for a simple connected graph with vertex set $V=\{1,2, \ldots, n\}$ and $\mathcal{V}$ denotes the space of the formal linear combinations of its vertices. The adjacencies in $\Gamma$, say $\{i, j\} \in E$, are denoted by $i \sim j$ and $\Gamma_{k}(i)=\{j \mid \partial(i, j)=k\}$ represents the set of vertices at distance $k$ from $i$, where $\partial(\cdot, \cdot)$ is the distance function in $\Gamma$. For simplicity we will write $\Gamma(i)$ instead of $\Gamma_{1}(i)$. Every vertex $i$ is associated to the $i$-th unitary (or characteristic) vector $\boldsymbol{e}_{i} \in \mathbb{R}^{n}$ and, consequently, $\mathcal{V}$ is identified with $\mathbb{R}^{n}$. With this identification in mind, the adjacency matrix $\boldsymbol{A}$ of $\Gamma$ can be seen as the matrix of an endomorphism in $\mathcal{V}$ with respect to the basis $\left\{\boldsymbol{e}_{i}\right\}_{i \in V}$.

The set of different eigenvalues of $\boldsymbol{A}$ is denoted by ev $\Gamma:=\left\{\lambda_{0}, \lambda_{1}, \ldots, \lambda_{d}\right\}$, where $\lambda_{0}>\lambda_{1}>\cdots>\lambda_{d}$, and the spectrum of $\Gamma$ is defined by

$$
\operatorname{sp} \Gamma:=\operatorname{sp} \boldsymbol{A}=\left\{\lambda_{0}^{m\left(\lambda_{0}\right)}, \lambda_{1}^{m\left(\lambda_{1}\right)}, \cdots, \lambda_{d}^{m\left(\lambda_{d}\right)}\right\},
$$

where $m\left(\lambda_{l}\right)$ stands for the multiplicity of the eigenvalue $\lambda_{l}$. From the Perron-Frobenius 
theorem for nonnegative matrices, we have that $\lambda_{0} \geqslant\left|\lambda_{d}\right|$ and equality is attained if and only if $\Gamma$ is a bipartite graph; see e.g. [1]. Moreover, $m\left(\lambda_{0}\right)=1$ and every non-null vector of $\operatorname{Ker}\left(\boldsymbol{A}-\lambda_{0} \boldsymbol{I}\right)$ has all its components either positive or negative. We denote by $\boldsymbol{\nu} \in \operatorname{Ker}\left(\boldsymbol{A}-\lambda_{0} \boldsymbol{I}\right)$ the unique positive eigenvector with minimum component equal to one. Let us remark that in the case of $\delta$-regular graphs we have that $\lambda_{0}=\delta$ and the vector $\boldsymbol{\nu}$ turns out to be the all-1 vector $\boldsymbol{j}$.

Note that $\mathcal{V}$ is a module over the quotient ring $\mathbb{R}[x] / \mathcal{I}$, where $\mathcal{I}$ is the ideal generated by the polynomial $Z=\prod_{l=0}^{d}\left(x-\lambda_{l}\right)$, which vanishes in $\boldsymbol{A}$, with product defined by

$$
p \boldsymbol{u}:=p(\boldsymbol{A}) \boldsymbol{u} \quad \text { for every } p \in \mathbb{R}[x] / \mathcal{I} \text { and } \boldsymbol{u} \in \mathcal{V} .
$$

Recall that, for every $0 \leqslant l \leqslant d$, the orthogonal projection $\boldsymbol{E}_{l}$ of $\mathcal{V}$ onto the eigenspace $\mathcal{E}_{l}=\operatorname{Ker}\left(\boldsymbol{A}-\lambda_{l} \boldsymbol{I}\right)$ can be written as

$$
\boldsymbol{E}_{l} \boldsymbol{u}=Z_{l} \boldsymbol{u}, \quad \boldsymbol{u} \in \mathcal{V}
$$

where $Z_{l}=\frac{(-1)^{l}}{\pi_{l}} \prod_{0 \leqslant h \leqslant d(h \neq l)}\left(x-\lambda_{l}\right)$ and $\pi_{l}:=\prod_{0 \leqslant h \leqslant d(h \neq l)}\left|\lambda_{h}-\lambda_{l}\right|$.

\section{The local spectrum of a vertex set and its antipodal}

Given a nonempty set $C$ of vertices of $\Gamma$, we consider the map $\rho: \mathcal{P}(V) \rightarrow \mathcal{V}$ defined by $\boldsymbol{\rho} \emptyset=\mathbf{0}$ and $\boldsymbol{\rho} C=\sum_{i \in C} \nu_{i} \boldsymbol{e}_{i}$ for $C \neq \emptyset$ and denote by $\boldsymbol{e}_{C}$ the normalized vector $\boldsymbol{\rho} C /\|\boldsymbol{\rho} C\|$. If $\boldsymbol{e}_{C}=\boldsymbol{z}_{C}\left(\lambda_{0}\right)+\boldsymbol{z}_{C}\left(\lambda_{1}\right)+\cdots+\boldsymbol{z}_{C}\left(\lambda_{d}\right)$ is the spectral decomposition of $\boldsymbol{e}_{C}$; that is $\boldsymbol{z}_{C}\left(\lambda_{l}\right)=\boldsymbol{E}_{l} \boldsymbol{e}_{C} \in \mathcal{E}_{l}, 0 \leqslant l \leqslant d$, the $C$-multiplicity (or $C$-local multiplicity) of the eigenvalue $\lambda_{l}$ is defined by $m_{C}\left(\lambda_{l}\right)=\left\|\boldsymbol{z}_{C}\left(\lambda_{l}\right)\right\|^{2}$. Note that, since

$$
\boldsymbol{z}_{C}\left(\lambda_{0}\right)=\boldsymbol{E}_{0} \boldsymbol{e}_{C}=\frac{1}{\|\boldsymbol{\rho} C\|} \frac{\langle\boldsymbol{\rho} C, \boldsymbol{\nu}\rangle}{\|\boldsymbol{\nu}\|^{2}} \boldsymbol{\nu}=\frac{1}{\|\boldsymbol{\rho} C\|} \sum_{i \in C} \nu_{i} \frac{\nu_{i}}{\|\boldsymbol{\nu}\|^{2}} \boldsymbol{\nu}=\frac{\|\boldsymbol{\rho} C\|}{\|\boldsymbol{\nu}\|^{2}} \boldsymbol{\nu},
$$

we get $m_{C}\left(\lambda_{0}\right)=\frac{\|\boldsymbol{\rho} C\|^{2}}{\|\boldsymbol{\nu}\|^{2}}$. Then, if $\mu_{0}\left(=\lambda_{0}\right), \mu_{1}, \ldots, \mu_{d_{C}}$ are the eigenvalues with non-zero $C$-multiplicity, the $C$-spectrum (or $C$-local spectrum) is defined by

$$
\operatorname{sp}_{C} \Gamma:=\left\{\mu_{0}^{m_{C}\left(\mu_{0}\right)}, \mu_{1}^{m_{C}\left(\mu_{1}\right)}, \ldots, \mu_{d_{C}}^{m_{C}\left(\mu_{d_{C}}\right)}\right\}
$$

with $\mu_{0}>\mu_{1}>\cdots>\mu_{d_{C}}$, and the set of different eigenvalues of $C$ is denoted by $\operatorname{ev}_{C} \Gamma:=\left\{\mu_{0}, \mu_{1}, \ldots, \mu_{d_{C}}\right\}$. Note that, since $\boldsymbol{e}_{C}$ is unitary, we have $\sum_{l=0}^{d_{C}} m_{C}\left(\lambda_{l}\right)=1$ or, equivalently, the vector

$$
\boldsymbol{m}_{C}=\left(\left\|\boldsymbol{z}_{C}\left(\mu_{0}\right)\right\|,\left\|\boldsymbol{z}_{C}\left(\mu_{1}\right)\right\|, \ldots,\left\|\boldsymbol{z}_{C}\left(\mu_{d_{C}}\right)\right\|\right) \in \mathbb{R}^{d_{C}+1},
$$

is also unitary. As we have done for the spectrum of $\Gamma$, in order to simplify notation we introduce the moment-like parameters

$$
\pi_{l}(C):=\prod_{0 \leqslant h \leqslant d_{C}(h \neq l)}\left|\mu_{h}-\mu_{l}\right| \quad\left(0 \leqslant l \leqslant d_{C}\right) .
$$


The set $\Gamma_{k}(C)=\{v \in V \mid \partial(v, C)=k\}$ of vertices at distance $k$ from $C$ is denoted by $C_{k}$. Thus, if $C$ has eccentricity $\varepsilon_{C}, C_{0}(=C), C_{1}, \ldots, C_{\varepsilon_{C}}$ is a partition of $V$. We denote by $\bar{C}$ the set $C_{\varepsilon_{C}}$ of vertices at maximum distance from $C$, and we refer to it as its antipodal set. If there is no possible confusion, we will write $D=\bar{C}$.

The polynomial $Z_{C}=\prod_{l=0}^{d_{C}}\left(x-\mu_{l}\right)$ is the monic polynomial with minimum degree such that $Z_{C} \boldsymbol{e}_{C}=\mathbf{0}$, and the polynomial

$$
H_{C}=\frac{\|\boldsymbol{\nu}\|^{2}}{\pi_{0}(C)\|\boldsymbol{\rho} C\|^{2}} \prod_{l=1}^{d_{C}}\left(x-\mu_{l}\right)
$$

satisfies $H_{C} \boldsymbol{\nu}=H_{C}\left(\lambda_{0}\right) \boldsymbol{\nu}=\frac{\|\boldsymbol{\nu}\|^{2}}{\|\boldsymbol{\rho} C\|^{2}} \boldsymbol{\nu}$. What is more, $H_{C}$ is the unique polynomial of degree at most $d_{C}$ satisfying

$$
H_{C} \boldsymbol{\rho} C=\frac{\|\boldsymbol{\rho} C\|^{2}}{\|\boldsymbol{\nu}\|^{2}} H_{C} \boldsymbol{\nu}=\boldsymbol{\nu}
$$

and so, inspired by Hoffman [8], it is named the C-local Hoffman polynomial. This allows us to conclude that the eccentricity of $C$ and the number of $C$-local eigenvalues are related by $\varepsilon_{C} \leqslant d_{C}$; see [5]. In case of equality, $\varepsilon_{C}=d_{C}$, we say that $C$ is extremal.

Proposition 2.1 Let $C$ be an extremal set and let $D$ be its antipodal set. Then, $\mathrm{ev}_{C} \Gamma \subset$ $\mathrm{ev}_{D} \Gamma$ and the $C$-multiplicities and D-multiplicities satisfy

$$
m_{C}\left(\mu_{l}\right) m_{D}\left(\mu_{l}\right) \geqslant \frac{\pi_{0}^{2}(C)}{\pi_{l}^{2}(C)} \frac{\|\boldsymbol{\rho} C\|^{2}\|\boldsymbol{\rho} D\|^{2}}{\|\boldsymbol{\nu}\|^{4}} \quad \text { for all } \mu_{l} \in \mathrm{ev}_{C} \Gamma,
$$

where equality is equivalent to the linear dependence of the vectors $\boldsymbol{z}_{C}\left(\mu_{l}\right)$ and $\boldsymbol{z}_{D}\left(\mu_{l}\right)$.

Proof. Consider the interpolating polynomials associated with the local spectrum of $C$ :

$$
Z_{l}^{C}=\frac{(-1)^{l}}{\pi_{l}(C)} \prod_{0 \leqslant h \leqslant d_{C}(h \neq l)}\left(x-\mu_{h}\right) \quad\left(0 \leqslant l \leqslant d_{C}\right),
$$

verifying $Z_{l}^{C}\left(\mu_{h}\right)=\delta_{l h}$. Since both $Z_{l}^{C}$ and $H_{C}$ have degree $d_{C}$ and their leading coefficients are, respectively, $\frac{(-1)^{l}}{\pi_{l}(C)}$ and $\frac{\|\boldsymbol{\nu}\|^{2}}{\pi_{0}(C)\|\boldsymbol{\rho} C\|^{2}}$, the polynomial

$$
T=\pi_{0}(C) \frac{\|\boldsymbol{\rho} C\|^{2}}{\|\boldsymbol{\nu}\|^{2}} H_{C}-(-1)^{l} \pi_{l}(C) Z_{l}^{C}
$$

has degree less than $d_{C}$. The extremal character of $C$ gives

$$
\left\langle\boldsymbol{\rho} C, Z_{l}^{C} \boldsymbol{\rho} D\right\rangle=\left\langle Z_{l}^{C} \boldsymbol{\rho} C, \boldsymbol{\rho} D\right\rangle=\frac{(-1)^{l}}{\pi_{l}(C)}\left\langle x^{d_{C}} \boldsymbol{\rho} C, \boldsymbol{\rho} D\right\rangle \neq 0 .
$$

In particular, $Z_{l}^{C} \boldsymbol{\rho} D \neq \mathbf{0}$. Moreover, if $\mu_{l} \in \mathrm{ev}_{C} \Gamma$,

$$
\begin{aligned}
\left\langle\boldsymbol{\rho} C, Z_{l}^{C} \boldsymbol{\rho} D\right\rangle & =\left\langle\boldsymbol{\rho} C, \sum_{h=0}^{d} Z_{l}^{C}\left(\lambda_{h}\right) \boldsymbol{E}_{h} \boldsymbol{\rho} D\right\rangle \\
& =\left\langle\boldsymbol{\rho} C, Z_{l}^{C}\left(\mu_{l}\right) \boldsymbol{E}_{l} \boldsymbol{\rho} D+\sum_{\lambda_{h} \notin \mathrm{ev}_{C} \Gamma} Z_{l}^{C}\left(\lambda_{h}\right) \boldsymbol{E}_{h} \boldsymbol{\rho} D\right\rangle \\
& =\left\langle\boldsymbol{\rho} C, \boldsymbol{E}_{l} \boldsymbol{\rho} D\right\rangle=\|\boldsymbol{\rho} D\|\left\langle\boldsymbol{\rho} C, \boldsymbol{z}_{D}\left(\mu_{l}\right)\right\rangle,
\end{aligned}
$$


and $\operatorname{ev}_{C} \Gamma \subset \mathrm{ev}_{D} \Gamma$.

Since $T$ has degree less than $d_{C}=\varepsilon_{C}$, the vectors $T \boldsymbol{e}_{C}$ and $\boldsymbol{e}_{D}$ are orthogonal, giving:

$$
\begin{aligned}
0=\left\langle T \boldsymbol{e}_{C}, \boldsymbol{e}_{D}\right\rangle & =\pi_{0}(C) \frac{\|\boldsymbol{\rho} C\|^{2}}{\|\boldsymbol{\nu}\|^{2}}\left\langle H_{C} \boldsymbol{e}_{C}, \boldsymbol{e}_{D}\right\rangle-(-1)^{l} \pi_{l}(C)\left\langle Z_{l}^{C} \boldsymbol{e}_{C}, \boldsymbol{e}_{D}\right\rangle \\
& =\pi_{0}(C) \frac{\|\boldsymbol{\rho} C\|}{\|\boldsymbol{\rho} D\|\|\boldsymbol{\nu}\|^{2}}\left\langle H_{C} \boldsymbol{\rho} C, \boldsymbol{\rho} D\right\rangle-(-1)^{l} \pi_{l}(C)\left\langle\boldsymbol{z}_{C}\left(\mu_{l}\right), \boldsymbol{e}_{D}\right\rangle \\
& =\pi_{0}(C) \frac{\|\boldsymbol{\rho} C\|}{\|\boldsymbol{\rho} D\|\|\boldsymbol{\nu}\|^{2}}\langle\boldsymbol{\nu}, \boldsymbol{\rho} D\rangle-(-1)^{l} \pi_{l}(C)\left\langle\boldsymbol{z}_{C}\left(\mu_{l}\right), \boldsymbol{z}_{D}\left(\mu_{l}\right)\right\rangle \\
& =\pi_{0}(C) \frac{\|\boldsymbol{\rho} C\|\|\boldsymbol{\rho} D\|}{\|\boldsymbol{\nu}\|^{2}}-(-1)^{l} \pi_{l}(C)\left\|\boldsymbol{z}_{C}\left(\mu_{l}\right)\right\|\left\|\boldsymbol{z}_{D}\left(\mu_{l}\right)\right\| \cos \alpha_{l}^{(C, D)}
\end{aligned}
$$

where $\alpha_{l}^{(C, D)}$ is the angle between the vectors $\boldsymbol{z}_{C}\left(\mu_{l}\right), \boldsymbol{z}_{D}\left(\mu_{l}\right)$. Therefore,

$$
\left\langle\boldsymbol{z}_{C}\left(\mu_{l}\right), \boldsymbol{z}_{D}\left(\mu_{l}\right)\right\rangle=(-1)^{l} \frac{\pi_{0}(C)}{\pi_{l}(C)} \frac{\|\boldsymbol{\rho} C\|\|\boldsymbol{\rho} D\|}{\|\boldsymbol{\nu}\|^{2}},
$$

and also:

$$
\frac{\pi_{0}^{2}(C)}{\pi_{l}^{2}(C)} \frac{\|\boldsymbol{\rho} C\|^{2}\|\boldsymbol{\rho} D\|^{2}}{\|\boldsymbol{\nu}\|^{4}}=m_{C}\left(\mu_{l}\right) m_{D}\left(\mu_{l}\right) \cos ^{2} \alpha_{l}^{(C, D)} \leqslant m_{C}\left(\mu_{l}\right) m_{D}\left(\mu_{l}\right),
$$

where the equality occurs if and only if $\alpha_{l}^{(C, D)}$ is 0 or $\pi$.

Proposition 2.2 Let $C$ be an extremal set, $\varepsilon_{C}=d_{C}$, and let $D$ be its antipodal set. Then, the following statements are equivalent:

(a) For every $\mu_{l} \in \mathrm{ev}_{C} \Gamma$, we have

$$
m_{C}\left(\mu_{l}\right) m_{D}\left(\mu_{l}\right)=\frac{\pi_{0}^{2}(C)}{\pi_{l}^{2}(C)} \frac{\|\boldsymbol{\rho} C\|^{2}\|\boldsymbol{\rho} D\|^{2}}{\|\boldsymbol{\nu}\|^{4}} .
$$

(b) The projection of the vector $\tilde{\boldsymbol{m}}_{D}=\left(\left\|\boldsymbol{z}_{D}\left(\mu_{0}\right)\right\|,\left\|\boldsymbol{z}_{D}\left(\mu_{1}\right)\right\|, \ldots,\left\|\boldsymbol{z}_{D}\left(\mu_{\varepsilon_{C}}\right)\right\|\right)$ over the vector $\boldsymbol{m}_{C}=\left(\left\|\boldsymbol{z}_{C}\left(\mu_{0}\right)\right\|,\left\|\boldsymbol{z}_{C}\left(\mu_{1}\right)\right\|, \ldots,\left\|\boldsymbol{z}_{C}\left(\mu_{\varepsilon_{C}}\right)\right\|\right)$ is

$$
\frac{\|\boldsymbol{\rho} C\|\|\boldsymbol{\rho} D\|}{\|\boldsymbol{\nu}\|^{2}} \sum_{l=0}^{\varepsilon_{C}} \frac{\pi_{0}(C)}{\pi_{l}(C)}
$$

or, equivalently,

$$
\left(\sum_{l=0}^{\varepsilon_{C}} m_{D}\left(\mu_{l}\right)\right) \cos ^{2} \alpha^{(C, D)}=\left(\sum_{l=0}^{\varepsilon_{C}} \frac{\pi_{0}(C)}{\pi_{l}(C)}\right)^{2} \frac{\|\boldsymbol{\rho} C\|^{2}\|\boldsymbol{\rho} D\|^{2}}{\|\boldsymbol{\nu}\|^{4}}
$$

where $\alpha^{(C, D)}$ is the angle between the two vectors. 
(c) There exists a polynomial $p \in \mathbb{R}_{\varepsilon_{C}}[x]$ such that

$$
\boldsymbol{\rho} D=p \boldsymbol{\rho} C+\boldsymbol{z}, \quad \text { where } \boldsymbol{z} \in \bigoplus_{\lambda_{l} \in \mathrm{ev}_{D}} \Gamma \backslash \mathrm{ev}_{C} \Gamma \mathcal{E}_{l} .
$$

(d) For every $\mu_{l} \in \mathrm{ev}_{C} \Gamma$, we have

$$
\frac{\|\boldsymbol{\rho} D\|^{2}}{\sum_{l=0}^{\varepsilon_{C}} m_{D}\left(\mu_{l}\right)}=\|\boldsymbol{\nu}\|^{2}\left(\sum_{l=0}^{\varepsilon_{C}} \frac{m_{C}\left(\mu_{0}\right) \pi_{0}^{2}(C)}{m_{C}\left(\mu_{l}\right) \pi_{l}^{2}(C)}\right)^{-1}
$$

Proof. By adding up for $l=0,1, \ldots, \varepsilon_{C}$ the inequalities given in Proposition 2.1 we obtain:

$$
\left\langle\boldsymbol{m}_{C}, \tilde{\boldsymbol{m}}_{D}\right\rangle=\left\|\tilde{\boldsymbol{m}}_{D}\right\| \cos \alpha^{(C, D)}=\sum_{l=0}^{\varepsilon_{C}}\left\|\boldsymbol{z}_{C}\left(\mu_{l}\right)\right\|\left\|\boldsymbol{z}_{D}\left(\mu_{l}\right)\right\| \geqslant \frac{\|\boldsymbol{\rho} C\|\|\boldsymbol{\rho} D\|}{\|\boldsymbol{\nu}\|^{2}} \sum_{l=0}^{\varepsilon_{C}} \frac{\pi_{0}(C)}{\pi_{l}(C)}
$$

giving the equivalence between $(a)$ and $(b)$.

Suppose that $(a)$ holds. Then, given $\mu_{l} \in \mathrm{ev}_{C} \Gamma$, the vectors $\boldsymbol{z}_{D}\left(\mu_{l}\right), \boldsymbol{z}_{C}\left(\mu_{l}\right)$ are proportional. More precisely, by $(4)$, there exist $\xi_{l}>0$ such that $\boldsymbol{z}_{D}\left(\mu_{l}\right)=(-1)^{l} \xi_{l} \boldsymbol{z}_{C}\left(\mu_{l}\right)$. Let $p$ be the unique polynomial in $\mathbb{R}_{\varepsilon_{C}}[x]$ such that $p\left(\mu_{l}\right)=(-1)^{l} \frac{\|\boldsymbol{\rho} D\|}{\|\boldsymbol{\rho} C\|} \xi_{l}$ for all $\mu_{l} \in \mathrm{ev}_{C} \Gamma$. We have

$$
\begin{aligned}
\boldsymbol{E}_{l} \boldsymbol{\rho} D & =\|\boldsymbol{\rho} D\| \boldsymbol{z}_{D}\left(\mu_{l}\right)=(-1)^{l}\|\boldsymbol{\rho} D\| \xi_{l} \boldsymbol{z}_{C}\left(\mu_{l}\right) \\
& =(-1)^{l} \frac{\|\boldsymbol{\rho} D\|}{\|\boldsymbol{\rho} C\|} \xi_{l} \boldsymbol{E}_{l} \boldsymbol{\rho} C=p\left(\mu_{l}\right) \boldsymbol{E}_{l} \boldsymbol{\rho} C=\boldsymbol{E}_{l} p \boldsymbol{\rho} C
\end{aligned}
$$

Thus the vector $\boldsymbol{z}=\boldsymbol{\rho} D-p \boldsymbol{\rho} C \in \bigoplus_{\lambda_{l} \in \mathrm{ev}_{D}} \Gamma \backslash \mathrm{ev}_{C} \Gamma \mathcal{E}_{l}$ and $(c)$ is obtained. Conversely, assuming that $(c)$ holds, by projecting onto the eigenspace of $\mu_{l}\left(\mu_{l} \in \mathrm{ev}_{C} \Gamma\right)$ we obtain $\|\boldsymbol{\rho} D\| \boldsymbol{z}_{D}\left(\mu_{l}\right)=p\left(\mu_{l}\right)\|\boldsymbol{\rho} C\| \boldsymbol{z}_{C}\left(\mu_{l}\right)$ and Proposition 2.1 gives $(a)$.

Finally we prove the equivalence between $(c)$ and $(d)$. The existence of the polynomial $p$ in $(c)$ is equivalent to the linear dependence of the vectors $\boldsymbol{z}_{D}\left(\mu_{l}\right)$ and $\boldsymbol{z}_{C}\left(\mu_{l}\right)$ for all $\mu_{l} \in \mathrm{ev}_{C} \Gamma$, and Proposition 2.1 ensures us that

$$
m_{C}\left(\mu_{l}\right) m_{D}\left(\mu_{l}\right)=\frac{\pi_{0}^{2}(C)}{\pi_{l}^{2}(C)} \frac{\|\rho C\|^{2}\|\rho D\|^{2}}{\|\boldsymbol{\nu}\|^{4}} \quad\left(0 \leqslant l \leqslant d_{C}\right)
$$

Hence, in this case,

$$
\sum_{l=0}^{\varepsilon_{C}} m_{D}\left(\mu_{l}\right)=\frac{\|\boldsymbol{\rho} C\|^{2}\|\boldsymbol{\rho} D\|^{2}}{\|\boldsymbol{\nu}\|^{4}} \sum_{l=0}^{\varepsilon_{C}} \frac{\pi_{0}^{2}(C)}{m_{C}\left(\mu_{l}\right) \pi_{l}^{2}(C)}=\frac{\|\boldsymbol{\rho} D\|^{2}}{\|\boldsymbol{\nu}\|^{2}} \sum_{l=0}^{\varepsilon_{C}} \frac{m_{C}\left(\mu_{0}\right) \pi_{0}^{2}(C)}{m_{C}\left(\mu_{l}\right) \pi_{l}^{2}(C)},
$$

and the proof is concluded.

Corollary 2.3 The polynomial p described in Proposition 2.2(c) satisfies the following properties: 
(a) The polynomial $p \in \mathbb{R}_{\varepsilon_{C}}[x]$ is unique, has degree $\varepsilon_{C}$ and all its roots are real, different, and interlace the eigenvalues $\mu_{0}, \mu_{1}, \ldots, \mu_{\varepsilon_{C}}$.

(b) The value of $p$ at $\mu_{0}$ is:

$$
p\left(\mu_{0}\right)=\frac{\|\boldsymbol{\rho} D\|^{2}}{\|\boldsymbol{\rho} C\|^{2}}=\frac{\|\boldsymbol{\nu}\|^{2}}{\|\boldsymbol{\rho} C\|^{2}}\left(\sum_{l=0}^{\varepsilon_{C}} m_{D}\left(\mu_{l}\right)\right)\left(\sum_{l=0}^{\varepsilon_{C}} \frac{m_{C}\left(\mu_{0}\right) \pi_{0}^{2}(C)}{m_{C}\left(\mu_{l}\right) \pi_{l}^{2}(C)}\right)^{-1} .
$$

(c) Given $q \in \mathbb{R}_{\varepsilon_{C}-1}[x]$, we have:

$$
\sum_{l=0}^{\varepsilon_{C}} m_{C}\left(\mu_{l}\right) p\left(\mu_{l}\right) q\left(\mu_{l}\right)=0 \quad \text { and } \quad \sum_{l=0}^{\varepsilon_{C}} m_{C}\left(\mu_{l}\right) p^{2}\left(\mu_{l}\right)=\left(\sum_{l=0}^{\varepsilon_{C}} m_{D}\left(\mu_{l}\right)\right) p\left(\mu_{0}\right) .
$$

Proof. (a) Using (4), the computation

$$
\begin{aligned}
(-1)^{l} \frac{\pi_{0}(C)}{\pi_{l}(C)} \frac{\|\boldsymbol{\rho} C\|\|\boldsymbol{\rho} D\|}{\|\boldsymbol{\nu}\|^{2}} & =\left\langle\boldsymbol{z}_{C}\left(\mu_{l}\right), \boldsymbol{z}_{D}\left(\mu_{l}\right)\right\rangle=\left\langle\boldsymbol{e}_{C}, \boldsymbol{E}_{l} \boldsymbol{e}_{D}\right\rangle \\
& =\frac{1}{\|\boldsymbol{\rho} C\|\|\boldsymbol{\rho} D\|}\left\langle\boldsymbol{\rho} C, \boldsymbol{E}_{l} \boldsymbol{\rho} D\right\rangle \\
& =\frac{1}{\|\boldsymbol{\rho} C\|\|\boldsymbol{\rho} D\|}\left\langle\boldsymbol{\rho} C, \boldsymbol{E}_{l} p \boldsymbol{\rho} C\right\rangle \\
& =\frac{1}{\|\boldsymbol{\rho} C\|\|\boldsymbol{\rho} D\|} p\left(\mu_{l}\right)\left\langle\boldsymbol{\rho} C, \boldsymbol{E}_{l} \boldsymbol{\rho} C\right\rangle \\
& =\frac{\|\boldsymbol{\rho} C\|}{\|\boldsymbol{\rho} D\|} p\left(\mu_{l}\right)\left\langle\boldsymbol{z}_{C}\left(\mu_{l}\right), \boldsymbol{z}_{C}\left(\mu_{l}\right)\right\rangle=m_{C}\left(\mu_{l}\right) \frac{\|\boldsymbol{\rho} C\|}{\|\boldsymbol{\rho} D\|} p\left(\mu_{l}\right),
\end{aligned}
$$

gives

$$
p\left(\mu_{l}\right)=(-1)^{l} \frac{\pi_{0}(C)}{m_{C}\left(\mu_{l}\right) \pi_{l}(C)} \frac{\|\boldsymbol{\rho} D\|^{2}}{\|\boldsymbol{\nu}\|^{2}} \quad \text { for all } \mu_{l} \in \mathrm{ev} C,
$$

thus, the polynomial $p \in \mathbb{R}_{\varepsilon_{C}}[x]$ is unique and the alternation of the sign over $\operatorname{ev}_{C} \Gamma$ guaranties that their roots interlace its elements.

(b) From Proposition 2.2(c) we get

$$
\|\boldsymbol{\rho} D\|^{2}=\langle p \boldsymbol{\rho} C, \boldsymbol{\nu}\rangle=\langle\boldsymbol{\rho} C, p \boldsymbol{\nu}\rangle=p\left(\mu_{0}\right)\langle\boldsymbol{\rho} C, \boldsymbol{\nu}\rangle=p\left(\mu_{0}\right)\|\boldsymbol{\rho} C\|^{2} .
$$

This, together with Proposition 2.2(d), gives the equalities.

(c) Using (b) and (6),

$$
\begin{aligned}
\sum_{l=0}^{\varepsilon_{C}} m_{C}\left(\mu_{l}\right) p^{2}\left(\mu_{l}\right) & =\sum_{l=0}^{\varepsilon_{C}} m_{C}\left(\mu_{l}\right) \frac{\pi_{0}^{2}(C)}{m_{C}^{2}\left(\mu_{l}\right) \pi_{l}^{2}(C)} \frac{\|\boldsymbol{\rho} D\|^{4}}{\|\boldsymbol{\nu}\|^{4}} \\
& =\frac{\|\boldsymbol{\rho} D\|^{4}}{\|\boldsymbol{\rho} C\|^{4}} \sum_{l=0}^{\varepsilon_{C}} \frac{m_{C}^{2}\left(\mu_{0}\right) \pi_{0}^{2}(C)}{m_{C}\left(\mu_{l}\right) \pi_{l}^{2}(C)} \\
& =\frac{\|\boldsymbol{\rho} D\|^{2}}{\|\boldsymbol{\rho} C\|^{2}} \sum_{l=0}^{\varepsilon_{C}} m_{D}\left(\mu_{l}\right)=\left(\sum_{l=0}^{\varepsilon_{C}} m_{D}\left(\mu_{l}\right)\right) p\left(\mu_{0}\right) .
\end{aligned}
$$


The polynomials $Z_{l}^{C}$ defined in (3) allow us to write every polynomial $q \in \mathbb{R}_{\varepsilon_{C}}[x]$ as $q=\sum_{l=0}^{\varepsilon_{C}} q\left(\mu_{l}\right) Z_{l}^{C}$. In particular, $\sum_{l=0}^{\varepsilon_{C}} \mu_{l}^{k} Z_{l}^{C}=x^{k}, 0 \leqslant k \leqslant \varepsilon_{C}$. Equating the coefficients of degree $\varepsilon_{C}$ we obtain

$$
\sum_{l=0}^{\varepsilon_{C}}(-1)^{l} \frac{\mu_{l}^{k}}{\pi_{l}(C)}=\delta_{k \varepsilon_{C}} \quad\left(0 \leqslant k \leqslant \varepsilon_{C}\right) .
$$

Then,

$$
\sum_{l=0}^{\varepsilon_{C}}(-1)^{l} \frac{q\left(\mu_{l}\right)}{\pi_{l}(C)}=0 \quad \text { for all } q \in \mathbb{R}_{\varepsilon_{C}-1}[x]
$$

and

$$
\sum_{l=0}^{\varepsilon_{C}} m_{C}\left(\mu_{l}\right) p\left(\mu_{l}\right) q\left(\mu_{l}\right)=\pi_{0}(C) \frac{\|\boldsymbol{\rho} D\|^{2}}{\|\boldsymbol{\nu}\|^{2}} \sum_{l=0}^{\varepsilon_{C}}(-1)^{l} \frac{q\left(\mu_{l}\right)}{\pi_{l}(C)}=0 .
$$

Corollary 2.4 Let $C \subset V$ be an extremal set with $\operatorname{sp}_{C} \Gamma=\left\{\mu_{0}, \mu_{1}, \ldots, \mu_{d_{C}}\right\}$ and let $D$ be its antipodal set. If the statements of Proposition 2.2 hold, then the angle between the vectors $\boldsymbol{m}_{C}=\left(\left\|\boldsymbol{z}_{C}\left(\mu_{0}\right)\right\|,\left\|\boldsymbol{z}_{C}\left(\mu_{1}\right)\right\|, \ldots,\left\|\boldsymbol{z}_{C}\left(\mu_{d_{C}}\right)\right\|\right)$ and $\tilde{\boldsymbol{m}}_{D}=\left(\left\|\boldsymbol{z}_{D}\left(\mu_{0}\right)\right\|,\left\|\boldsymbol{z}_{D}\left(\mu_{1}\right)\right\|, \ldots\right.$, $\left.\left\|\boldsymbol{z}_{D}\left(\mu_{d_{C}}\right)\right\|\right)$ satisfies

$$
\cos \alpha^{(C, D)}=\frac{\sum_{l=0}^{\varepsilon_{C}} \frac{1}{\pi_{l}(C)}}{\sqrt{\sum_{l=0}^{\varepsilon_{C}} \frac{1}{m_{C}\left(\mu_{l}\right) \pi_{l}^{2}(C)}}} .
$$

\section{Completely pseudo-regular codes in combinatorial sense}

The notion of pseudo-distance-regularity was first introduced in [7] as a generalization for non-regular graphs of the distance-regularity. More precisely, in this section we are interested in $C$-local pseudo-distance-regularity, which, when restricted to regular graphs, is equivalent to the fact that $C$ is a completely regular code. For a more exhaustive study of this property see [5], where the authors obtain several characterizations which, in particular, yield new characterizations for completely regular codes.

Given a set $C$ of vertices of a graph $\Gamma$, with eccentricity $\varepsilon_{C}$, we associate to it the functions $a, b, c: V \longrightarrow\left[0, \lambda_{0}\right]$ defined for $i \in C_{k}$ by

$$
\begin{aligned}
& c(i)= \begin{cases}0 & (k=0) ; \\
\frac{1}{\nu_{i}} \sum_{j \in \Gamma(i) \cap C_{k-1}} \nu_{j} & \left(1 \leqslant k \leqslant \varepsilon_{C}\right) .\end{cases} \\
& a(i)=\frac{1}{\nu_{i}} \sum_{j \in \Gamma(i) \cap C_{k}} \nu_{j} \quad\left(0 \leqslant k \leqslant \varepsilon_{C}\right) .
\end{aligned}
$$




$$
b(i)= \begin{cases}\frac{1}{\nu_{i}} \sum_{j \in \Gamma(i) \cap C_{k+1}} \nu_{j} & \left(0 \leqslant k \leqslant \varepsilon_{C}-1\right) ; \\ 0 & \left(k=\varepsilon_{C}\right) .\end{cases}
$$

Since $\boldsymbol{\nu}$ is an eigenvector of eigenvalue $\lambda_{0}$,

$$
c(i)+a(i)+b(i)=\frac{1}{\nu_{i}} \sum_{j \in \Gamma(i)} \nu_{j}=\lambda_{0} \quad \text { for all } i \in V
$$

that is, the sum over the three functions $a, b, c$, is constant and their images are all in $\left[0, \lambda_{0}\right]$. In other words, by assigning weight $\nu_{i}$ to each vertex $i$, the average weighted degree becomes constant and the graph becomes "regularized". Note that, since every vertex in $C_{k}$ must be adjacent to a vertex of $C_{k-1}$, the function $c$ is strictly positive over $V \backslash C_{0}$. We say that $C$ is a flowing set when the associated function $b$ is strictly positive over $V \backslash C_{\varepsilon_{C}}$.

Lemma 3.1 Let $C \in V$ be a set of vertices with eccentricity $\varepsilon_{C}$ and let $D$ be its antipodal set. Then, $C$ is a flowing set if and only if $\varepsilon_{C}=\varepsilon_{D}=\varepsilon$ and the corresponding distance partitions, $C_{0}(=C), C_{1}, \ldots, C_{\varepsilon}$ and $D_{0}(=D), D_{1}, \ldots, D_{\varepsilon}$, satisfy $D_{k}=C_{\varepsilon-k}, 0 \leqslant k \leqslant \varepsilon$.

Proof. The condition suffices to guaranty that $C$ is a flowing set since it implies that the function $b$ corresponding to $C$ coincides with the function $c$ corresponding to $D$. Conversely, if $C$ is a flowing set, every vertex in $C_{k}$ is at distance $\varepsilon_{C}-k$ from $D$ and then $C_{k} \subset D \varepsilon_{C}-k, 0 \leqslant k \leqslant \varepsilon_{C}$. From this we get

$$
V=C_{0} \cup C_{1} \cup \cdots \cup C_{\varepsilon_{C}} \subset D_{\varepsilon_{C}} \cup D_{\varepsilon_{C}-1} \cup \cdots \cup D_{0} \subset V
$$

and, since $C_{k}$ (respectively, $\left.D_{k}\right), 1 \leqslant k \leqslant \varepsilon_{C}$, do not intersect each other, $\varepsilon_{C}=\varepsilon_{D}=\varepsilon$ and $D_{k}=C_{\varepsilon-k}, 0 \leqslant k \leqslant \varepsilon$.

Note that, by symmetry, the previous lemma establishes that $C$ is a flowing set if and only if $D$ is.

Definition 3.2 A graph $\Gamma$ is $C$-local pseudo-distance-regular (or pseudo-distance-regular around $C$ ) in combinatorial sense when the functions $c$, $a$ and $b$ associated to $C$ are constant over every $C_{k}, 0 \leqslant k \leqslant \varepsilon_{C}$. In this case we say that $C$ is a completely pseudoregular code.

In the sequel we will refer to this property by $C$-local pseudo-distance regularity when we want to emphasize the regularity of the graph, and we will use completely pseudoregular code when we focus our attention on the set of vertices $C$.

This definition generalizes, for any graph, the concept of completely regular code in a regular (or distance-regular) graph, where the above conditions on the fuctions $c, a, b$ imply that $C_{0}, C_{1}, \ldots, C_{\varepsilon_{C}}$ is a regular partition of $V$. 
Its clear that if $C$ is a completely pseudo-regular code in combinatorial sense, then $C$ is a flowing set. In this case, from Lemma 3.1 we have that $\bar{D}=C$ and the distance partitions associated to $C$ and $D$ coincide. Therefore, $D$ is also a completely pseudoregular code with the roles of the functions $b$ and $c$ interchanged.

For a completely pseudo-regular code $C$, we indicate by $c_{k}, a_{k}$ and $b_{k}$ the (constant) values of $c, a$ and $b$, respectively, over every vertex of $C_{k}$, and we refer to them as the pseudo-intersection numbers of $C$. Note that when $\Gamma$ is a regular graph and $C$ consists of a single vertex, the above numbers become the usual intersection numbers.

\subsection{Some characterizations of completely pseudo-regular codes}

In [5], several quasi-spectral characterizations of $C$-local pseudo-distance-regularity are given. The authors obtain their results through a sequence of orthogonal polynomials constructed from the $C$-local spectrum. In order to introduce these polynomials, let us first define, in the quotient ring $\mathbb{R}[x] /\left(Z_{C}\right)$, the following $C$-local scalar product:

$$
\langle p, q\rangle_{C}:=\left\langle p \boldsymbol{e}_{C}, q \boldsymbol{e}_{C}\right\rangle=\sum_{l=0}^{d_{C}} m_{C}\left(\mu_{l}\right) p\left(\mu_{l}\right) q\left(\mu_{l}\right) .
$$

A family of polynomials $r_{0}, r_{1}, \ldots, r_{d_{C}}$ is an orthogonal system with respect to the $C$-local scalar product when $\operatorname{deg} r_{k}=k$ and $\left\langle r_{k}, r_{h}\right\rangle_{C}=\delta_{k h}, 0 \leqslant k, h \leqslant d_{C}$. Then, the family of $C$-local predistance polynomials, $\left\{p_{k}^{C}\right\}_{0 \leqslant k \leqslant d_{C}}$ is the unique orthogonal system with respect to the $C$-local scalar product such that $\left\|p_{k}^{C}\right\|_{C}^{2}=p_{k}^{C}\left(\lambda_{0}\right), 0 \leqslant k \leqslant d_{C}$; see [2].

As mentioned, several characterizations of $C$-local pseudo-distance-regularity can be obtained in terms of these polynomials which, in this case, are called the $C$-local distance polynomials; see [5].

Theorem 3.3 A graph $\Gamma=(V, E)$ is $C$-local pseudo-distance-regular around a set $C \subset V$, with eccentricity $\varepsilon_{C}$, if and only if there exist a sequence of polynomials $r_{0}, r_{1}, \ldots, r_{\varepsilon_{C}}$, with $\operatorname{deg} r_{k}=k$, such that $\rho C_{k}=r_{k} \rho C$ for any $0 \leqslant k \leqslant \varepsilon_{C}$. Moreover, in this case, $\varepsilon_{C}=d_{C}$ and the polynomials $\left\{r_{k}\right\}_{0 \leqslant k \leqslant d_{C}}$ are the $C$-local (pre) distance polynomials.

Furthermore, for an extremal set $C$, the $C$-local pseudo-distance-regularity can be characterized in terms of only the highest degree $C$-local predistance polynomial.

Theorem 3.4 Let $\Gamma=(V, E)$ be a graph containing an extremal set $C \subset V, \varepsilon_{C}=d_{C}$, with antipodal set $\bar{C}$. Then $\Gamma$ is $C$-local pseudo-distance-regular in combinatorial sense if and only if any of the two following conditions holds:

(a) $p_{\varepsilon_{C}}^{C} \rho C=\rho \bar{C}$.

(b) $p_{d_{C}}^{C}\left(\lambda_{0}\right)=\frac{\|\boldsymbol{\rho} \bar{C}\|^{2}}{\|\boldsymbol{\rho} C\|^{2}}$.

In the next section, new proofs of the above two theorems will be provided by using an algebraic (or Terwilliger-like) approach to completely pseudo-regular codes. 


\section{Completely pseudo-regular codes in algebraic sense}

Let $C \subset V$ be a set of vertices of a simple connected graph $\Gamma=(V, E)$. For each $0 \leqslant k \leqslant \varepsilon_{C}$, let $\mathcal{E}_{k}^{\star}$ be the vector space having $\left\{\boldsymbol{e}_{i}\right\}_{i \in C_{k}}$ as a basis. Denote by $\boldsymbol{E}_{k}^{*}$ the projection $\mathcal{V} \rightarrow \mathcal{E}_{k}^{\star}$, so that $\mathcal{E}_{k}^{\star}=\boldsymbol{E}_{k}^{*} \mathcal{V}$ and $\boldsymbol{\rho} C_{k}=\boldsymbol{E}_{k}^{*} \boldsymbol{\nu}, 0 \leqslant k \leqslant \varepsilon_{C}$. As a generalization of the subconstituent algebras defined in [11], also known as Terwilliger algebras, we consider the algebra $\mathcal{T}_{C}$ generated by the linear operators $\boldsymbol{A}, \boldsymbol{E}_{0}^{*}, \boldsymbol{E}_{1}^{*}, \ldots, \boldsymbol{E}_{\varepsilon_{C}}^{*}$. A $\mathcal{T}_{C}$-module $W$ is a subspace of $\mathcal{V}$ which is invariant under the action of $\mathcal{T}_{C}$, that is, $\mathcal{T}_{C} W=W$.

In the context of association schemes, Terwilliger [11] defined a thin module as a $\mathcal{T}_{C^{-}}$ module $W$ satisfying $\operatorname{dim} \boldsymbol{E}_{k}^{*} W \leqslant 1$ for every $k$. As commented in [3, 5], if we consider a single vertex $i$, the notion of $\{i\}$-local pseudo-distance-regularity is equivalent to the thin character of the primary $\mathcal{T}_{\{i\}}$-module, that is, the unique irreducible module containing $\boldsymbol{\rho}\{i\}=\nu_{i} \boldsymbol{e}_{i}$. With the aim of generalizing this definition to any subset of vertices, let us consider a vector $\boldsymbol{w}_{C} \in \mathcal{E}_{0}^{\star}$ and $W_{C}:=\mathcal{T}_{C} \boldsymbol{w}_{C} \subset \mathcal{V}$, the minimum $\mathcal{T}_{C}$-module containing $\boldsymbol{w}_{C}$. The definition of completely pseudo-regular code (or $C$-local pseudo-distance-regularity) in algebraic sense will require the subspaces $\boldsymbol{E}_{k}^{*} W_{C}, 0 \leqslant k \leqslant d_{C}$, to be one-dimensional. Let us first study some conditions that $\boldsymbol{w}_{C}$ must satisfy. Let $\boldsymbol{w}_{C}=\sum_{i \in C} \xi_{i} \boldsymbol{e}_{i}$. Since $\boldsymbol{E}_{k}=\frac{(-1)^{k}}{\pi_{k}} \prod_{0 \leqslant l \leqslant d(l \neq k)}\left(\boldsymbol{A}-\lambda_{l} \boldsymbol{I}\right) \in \mathcal{T}_{C}$ for each $0 \leqslant k \leqslant d_{C}$, we have

$$
\begin{aligned}
\boldsymbol{E}_{k}^{*} \boldsymbol{E}_{0} \boldsymbol{w}_{C} & =\sum_{i \in C} \xi_{i} \boldsymbol{E}_{k}^{*} \boldsymbol{E}_{0}\left(\frac{\nu_{i}}{\|\boldsymbol{\nu}\|^{2}} \boldsymbol{\nu}+\boldsymbol{z}_{i}\left(\lambda_{1}\right)+\cdots+\boldsymbol{z}_{i}\left(\lambda_{d}\right)\right) \\
& =\sum_{i \in C} \frac{\xi_{i} \nu_{i}}{\|\boldsymbol{\nu}\|^{2}} \boldsymbol{E}_{k}^{*} \boldsymbol{\nu}=\left(\sum_{i \in C} \frac{\xi_{i} \nu_{i}}{\|\boldsymbol{\nu}\|^{2}}\right) \boldsymbol{\rho} C_{k} .
\end{aligned}
$$

Thus if $\operatorname{dim} \boldsymbol{E}_{k}^{*} W_{C}=1$, the vector $\boldsymbol{\rho} C_{k}$ will constitute a basis of $\boldsymbol{E}_{k}^{*} W_{C}$. In particular, $\boldsymbol{w}_{C}=\boldsymbol{E}_{0}^{*} \boldsymbol{w}_{C}$ is linearly dependent with $\boldsymbol{\rho} C_{0}$. Thus, the generalization for a set of vertices of the definition of Terwilliger for a single vertex must be:

Definition 4.1 A set of vertices $C \subset V$ of a graph $\Gamma$ is a completely pseudo-regular code in algebraic sense when $\operatorname{dim} \boldsymbol{E}_{k}^{*} W_{C}=1$ for every $0 \leqslant k \leqslant \varepsilon_{C}$, where $W_{C}$ is the $\mathcal{T}_{C}$-module $W_{C}:=\mathcal{T}_{C} \boldsymbol{\rho} C=\mathcal{T}_{C} \boldsymbol{E}_{k}^{*} \boldsymbol{\nu}$.

This definition generalizes also, for any graph, the one given in [10] for a set of vertices in a distance-regular graph.

From the previous comments, if $C$ is a completely pseudo-regular code in algebraic sense, then $\boldsymbol{E}_{k}^{*} T \boldsymbol{\rho} C \in \operatorname{span}\left\{\boldsymbol{\rho} C_{k}\right\}$ for every $T \in \mathcal{T}_{C}$ and $0 \leqslant k \leqslant \varepsilon_{C}$. The following result gives a characterization of completely pseudo-regular codes in algebraic sense, which coincides with the one of Theorem 3.3. This proves the equivalence between combinatorial and algebraic approaches to completely pseudo-regular codes. So, once proved, we speak indistinctly of one or another concept.

Theorem 4.2 A set of vertices $C \subset V$ of a graph $\Gamma$ is a completely pseudo-regular code in algebraic sense if and only if there exist polynomials $p_{0}, p_{1}, \ldots, p_{\varepsilon_{C}}$ in $\mathbb{R}_{\varepsilon_{C}}[x]$ such that $p_{k} \boldsymbol{\rho} C=\boldsymbol{\rho} C_{k}, 0 \leqslant k \leqslant \varepsilon_{C}$. 
Proof. Suppose that $C$ is a completely pseudo-regular code in algebraic sense. Given $r \in \mathbb{R}_{\varepsilon_{C}}[x]$ and $0 \leqslant k \leqslant \varepsilon_{C}$, consider $\xi_{k}(r) \in \mathbb{R}$ such that $\boldsymbol{E}_{k}^{*} r \boldsymbol{\rho} C=\xi_{k}(r) \boldsymbol{\rho} C_{k}$. We have that the map

$$
\mathbb{R}_{\varepsilon_{C}}[x] \stackrel{\Theta}{\longrightarrow} \mathbb{R}^{\varepsilon_{C}+1} \quad \text { defined by } \quad \Theta r:=\left(\xi_{0}(r), \xi_{1}(r), \ldots, \xi_{\varepsilon_{C}}(r)\right)
$$

is linear. If $r \in \mathbb{R}_{\varepsilon_{C}}[x]$ satisfies $\Theta r=\mathbf{0}$ then $\boldsymbol{E}_{k}^{*} r \boldsymbol{\rho} C=\mathbf{0}$ for every $k$ and $r \boldsymbol{\rho} C=$ $\left(\sum_{k=0}^{\varepsilon_{C}} \boldsymbol{E}_{k}^{*}\right) r \boldsymbol{\rho} C=\mathbf{0}$. Consequently, $r$ will vanish over all the $d_{C}+1$ elements of $\operatorname{ev}_{C} \Gamma$, and, since $\operatorname{deg} r \leqslant \varepsilon_{C} \leqslant d_{C}$, we conclude that $r=0$. This proves that $\Theta$ is an isomorphism, and by considering the polynomial $p_{k} \in \mathbb{R}_{\varepsilon_{C}}[x]$ such that

$$
\Theta p_{k}=(0, \ldots, \stackrel{(k)}{1}, \ldots, 0),
$$

we have that $p_{k} \rho C=\rho C_{k}, 0 \leqslant k \leqslant \varepsilon_{C}$.

Conversely, let us now show that the existence of such polynomials implies that $C$ is a completely pseudo-regular code. With this aim, consider the polynomial $q=p_{0}+p_{1}+$ $\cdots+p_{\varepsilon_{C}} \in \mathbb{R}_{\varepsilon_{C}}[x]$ satisfying $q \boldsymbol{\rho} C=\sum_{k=0}^{\varepsilon_{C}} \boldsymbol{\rho} C_{k}=\boldsymbol{\nu}$. Thus, $q\left(\mu_{0}\right)=\frac{\|\boldsymbol{\nu}\|^{2}}{\|\boldsymbol{\rho} C\|^{2}}$ and $q\left(\mu_{l}\right)=0$, $l=1, \ldots, d_{C}$, giving $d_{C} \leqslant \operatorname{deg} q \leqslant \varepsilon_{C} \leqslant d_{C}$, so that $C$ is extremal $\left(\varepsilon_{C}=d_{C}\right)$. Moreover, $q=\frac{\|\boldsymbol{\nu}\|^{2}}{\pi_{0}(C)\|\boldsymbol{\rho} C\|^{2}}\left(x-\mu_{1}\right) \cdots\left(x-\mu_{\varepsilon_{C}}\right)$ is the $C$-local Hoffman polynomial $H_{C}$ defined in (1).

The hypothesis guaranties that the polynomials $p_{k}, 0 \leqslant k \leqslant \varepsilon_{C}$, constitute a basis of $\mathbb{R}_{\varepsilon_{C}}[x]$, identified with $\mathbb{R}[x] /\left(Z_{C}\right)$. Define $\gamma_{h k}^{l} \in \mathbb{R}$ by

$$
p_{h} p_{k}=\sum_{l=0}^{\varepsilon_{C}} \gamma_{h k}^{l} p_{l} \quad\left(0 \leqslant h, k \leqslant \varepsilon_{C}\right) .
$$

Every element of $\boldsymbol{E}_{k}^{*} \mathcal{T}_{C} \boldsymbol{\rho} C$ can be seen as a linear combination of vectors $T_{r} T_{r-1} \cdots T_{1} \boldsymbol{\rho} C$, where $T_{l}=\boldsymbol{E}_{t_{l}}^{*} p_{s_{l}}, 1 \leqslant l \leqslant r$ and $t_{r}=k$. We can suppose that $s_{1}=t_{1}$ (since, otherwise, we get the zero vector). Then,

$$
\begin{aligned}
T_{1} \boldsymbol{\rho} C & =\boldsymbol{E}_{t_{1}}^{*} p_{s_{1}} \boldsymbol{\rho} C=\boldsymbol{E}_{t_{1}}^{*} \boldsymbol{\rho} C_{s_{1}}=\boldsymbol{\rho} C_{s_{1}}=p_{s_{1}} \boldsymbol{\rho} C, \\
T_{2} T_{1} \boldsymbol{\rho} C & =\boldsymbol{E}_{t_{2}}^{*} p_{s_{2}} p_{s_{1}} \boldsymbol{\rho} C=\boldsymbol{E}_{t_{2}}^{*}\left(\sum_{l=0}^{\varepsilon_{C}} \gamma_{s_{2} s_{1}}^{l} p_{l}\right) \boldsymbol{\rho} C=\boldsymbol{E}_{t_{2}}^{*} \sum_{l=0}^{\varepsilon_{C}} \gamma_{s_{2} s_{1}}^{l} \boldsymbol{\rho} C_{l}=\gamma_{t_{1} s_{2}}^{t_{2}} \boldsymbol{\rho} C_{t_{2}} \\
& =\gamma_{t_{1} s_{2}}^{t_{2}} p_{t_{2}} \boldsymbol{\rho} C
\end{aligned}
$$

and, iterating, we get

$$
T_{r} \cdots T_{1} \rho C=\gamma_{t_{1} s_{2}}^{t_{2}} \cdots \gamma_{t_{r-1} s_{r}}^{t_{r}} p_{t_{r}} \rho C=\gamma_{t_{1} s_{2}}^{t_{2}} \cdots \gamma_{t_{r-1} s_{r}}^{t_{r}} \rho C_{k} .
$$

Hence, $\operatorname{dim} \boldsymbol{E}_{k}^{*} W_{C}=1,0 \leqslant k \leqslant \varepsilon_{C}$, and $C$ is a completely pseudo-regular code in algebraic sense.

In particular, notice that we have shown that the condition of being extremal, $\varepsilon_{C}=d_{C}$, is necessary for being a completely pseudo-regular code. Moreover, the polynomials of Theorem 4.2 satisfy the following properties: 
Corollary 4.3 Let $\Gamma=(V, E)$ be a graph and $C \subset V$ a completely pseudo-regular code. For every $0 \leqslant k \leqslant \varepsilon_{C}\left(=d_{C}\right)$, the polynomial $p_{k} \in \mathbb{R}_{\varepsilon_{C}}[x]$ satisfying $p_{k} \boldsymbol{\rho} C=\boldsymbol{\rho} C_{k}$ is unique, it has degree $k$, and coincides with the $C$-local predistance polynomial, $p_{k}=p_{k}^{C}$.

Proof. The unicity is provided by the fact that the map $\Theta$ defined in (8) is an isomorphism. In particular, this gives that $p_{0}=1$. Now, consider $1 \leqslant k \leqslant d_{C}$, if $\operatorname{deg} p_{k}<k$ a contradiction arises: $\left\|\boldsymbol{\rho} C_{k}\right\|^{2}=\left\langle p_{k} \boldsymbol{\rho} C, \boldsymbol{\rho} C_{k}\right\rangle=0$. Let $s, 1 \leqslant s \leqslant \varepsilon_{C}-1$, be the maximum integer such that $\operatorname{deg} p_{s}>s$. There exist $\xi_{s+1}, \ldots, \xi_{\varepsilon_{C}} \in \mathbb{R}$ such that the polynomial $q=p_{s}+\xi_{s+1} p_{s+1}+\cdots+\xi_{\varepsilon_{C}} p_{\varepsilon_{C}}$ has degree at most $s$. Consider $l \geqslant s+1$ such that $\xi_{l} \neq 0$. Then,

$$
\begin{aligned}
\left\langle q \rho C, \boldsymbol{\rho} C_{l}\right\rangle & =\left\langle p_{s} \boldsymbol{\rho} C, \boldsymbol{\rho} C_{l}\right\rangle+\sum_{h=s+1}^{\varepsilon_{C}} \xi_{h}\left\langle p_{h} \boldsymbol{\rho} C, \boldsymbol{\rho} C_{l}\right\rangle \\
& =\left\langle\boldsymbol{\rho} C_{s}, \boldsymbol{\rho} C_{l}\right\rangle+\sum_{h=s+1}^{\varepsilon_{C}} \xi_{h}\left\langle\boldsymbol{\rho} C_{h}, \boldsymbol{\rho} C_{l}\right\rangle=\xi_{l}\left\|\boldsymbol{\rho} C_{l}\right\|^{2} \neq 0
\end{aligned}
$$

On the other hand, since $\operatorname{deg} q \leqslant s<s+1 \leqslant l$, we get $\left\langle q \rho C, \rho C_{l}\right\rangle=0$, which is impossible. So it does not exists such an index $s$ and $\operatorname{deg} p_{k}=k$ for every $0 \leqslant k \leqslant \varepsilon_{C}$. Finally, the polynomials $\left\{p_{k}\right\}_{0 \leqslant k \leqslant \varepsilon_{C}}$ are orthogonal:

$$
\left\langle p_{k}, p_{h}\right\rangle_{C}=\left\langle p_{k} \boldsymbol{e}_{C}, p_{h} \boldsymbol{e}_{C}\right\rangle=\frac{1}{\|\boldsymbol{\rho} C\|^{2}}\left\langle\boldsymbol{\rho} C_{k}, \boldsymbol{\rho} C_{h}\right\rangle=0 \quad \text { for } k \neq h,
$$

and they have norm:

$$
\begin{aligned}
\left\|p_{k}\right\|_{C}^{2} & =\frac{1}{\|\boldsymbol{\rho} C\|^{2}}\left\langle p_{k} \boldsymbol{\rho} C, p_{k} \boldsymbol{\rho} C\right\rangle=\frac{1}{\|\boldsymbol{\rho} C\|^{2}}\left\langle\boldsymbol{\rho} C_{k}, \boldsymbol{\rho} C_{k}\right\rangle \\
& =\frac{1}{\|\boldsymbol{\rho} C\|^{2}}\left\langle\boldsymbol{\nu}, p_{k} \boldsymbol{\rho} C\right\rangle=\frac{1}{\|\boldsymbol{\rho} C\|^{2}}\left\langle p_{k} \boldsymbol{\nu}, \boldsymbol{\rho} C\right\rangle=\frac{p_{k}\left(\mu_{0}\right)}{\|\boldsymbol{\rho} C\|^{2}}\langle\boldsymbol{\nu}, \boldsymbol{\rho} C\rangle=p_{k}\left(\mu_{0}\right) .
\end{aligned}
$$

Consequently, they are the $C$-local predistance polynomials $\left\{p_{k}^{C}\right\}_{0 \leqslant k \leqslant d_{C}}$, as claimed.

The following result gives another characterization of completely pseudo-regular codes, which is proved by using the algebraic approach.

Theorem 4.4 Let $\Gamma=(V, E)$ be a connected graph with vertex subset $C \subset V$ having eccentricity $\varepsilon_{C}$ and local eigenvalues $\mathrm{ev}_{C} \Gamma=\left\{\mu_{0}, \mu_{1}, \ldots, \mu_{d_{C}}\right\}$. Let us consider the distance partition $V=C_{0} \cup C_{1} \cup \cdots \cup C_{\varepsilon_{C}}$ given by the distance to $C$, and the spectral decomposition $\boldsymbol{\rho} C=\hat{\boldsymbol{z}}_{C}\left(\mu_{0}\right)+\hat{\boldsymbol{z}}_{C}\left(\mu_{1}\right)+\cdots+\hat{\boldsymbol{z}}_{C}\left(\mu_{d_{C}}\right)$. Then, $C$ is a completely pseudo-regular code if and only if the subspaces $R, S \subset \mathcal{V}$ generated respectively by $\rho C_{0}, \rho C_{1}, \ldots, \rho C_{\varepsilon_{C}}$ and $\hat{\boldsymbol{z}}_{C}\left(\mu_{0}\right), \hat{\boldsymbol{z}}_{C}\left(\mu_{1}\right), \ldots, \hat{\boldsymbol{z}}_{C}\left(\mu_{d_{C}}\right)$ coincide. That is, with Terwilliger's notation ([11, 12]),

$$
R=\operatorname{span}\left\{\boldsymbol{E}_{0}^{*} \boldsymbol{\nu}, \boldsymbol{E}_{1}^{*} \boldsymbol{\nu}, \ldots, \boldsymbol{E}_{\varepsilon_{C}}^{*} \boldsymbol{\nu}\right\}=S=\operatorname{span}\left\{\boldsymbol{E}_{0} \boldsymbol{E}_{0}^{*} \boldsymbol{\nu}, \boldsymbol{E}_{1} \boldsymbol{E}_{0}^{*} \boldsymbol{\nu}, \ldots, \boldsymbol{E}_{d_{C}} \boldsymbol{E}_{0}^{*} \boldsymbol{\nu}\right\} .
$$


Proof. First, notice that, since the involved vectors are linearly independent we have $\operatorname{dim} R=\varepsilon_{C}$ and $\operatorname{dim} S=d_{C}$. Suppose that $C$ is a completely pseudo-regular code. Then, Theorem 4.2 guaranties that $C$ is extremal, $d_{C}=\varepsilon_{C}$, and there exist polynomials $p_{0}, p_{1}, \ldots, p_{\varepsilon_{C}}$ in $\mathbb{R}_{\varepsilon_{C}}[x]$ such that $p_{k} \boldsymbol{\rho} C=\boldsymbol{\rho} C_{k}, 0 \leqslant k \leqslant \varepsilon_{C}$. Given $h, 0 \leqslant h \leqslant \varepsilon_{C}$, we have

$$
\hat{\boldsymbol{z}}_{C}\left(\mu_{h}\right)=\boldsymbol{E}_{h} \boldsymbol{\rho} C=\left(\sum_{k=0}^{\varepsilon_{C}} \boldsymbol{E}_{k}^{*}\right) \boldsymbol{E}_{h} \boldsymbol{\rho} C=\sum_{k=0}^{\varepsilon_{C}} \boldsymbol{E}_{k}^{*} \boldsymbol{E}_{h} \boldsymbol{\rho} C=\sum_{k=0}^{\varepsilon_{C}} a_{h k} \boldsymbol{\rho} C_{k},
$$

where $a_{h k} \in \mathbb{R}$, thus $\hat{\boldsymbol{z}}_{C}\left(\mu_{h}\right) \in S$ and $R=S$.

Suppose now that $R=S$. In particular, $\varepsilon_{C}=d_{C}$ and $C$ is extremal. For every $0 \leqslant k \leqslant \varepsilon_{C}$, there are $b_{k h} \in \mathbb{R}, 0 \leqslant h \leqslant \varepsilon_{C}$, satisfying

$$
\boldsymbol{\rho} C_{k}=\sum_{h=0}^{\varepsilon_{C}} b_{k h} \hat{\boldsymbol{z}}_{C}\left(\mu_{h}\right) \text {. }
$$

Define $p_{k} \in \mathbb{R}_{\varepsilon_{C}}[x]$ as the unique polynomial such that $p_{k}\left(\mu_{h}\right)=b_{k h}$ for every $0 \leqslant h \leqslant \varepsilon_{C}$. Then

$$
\boldsymbol{\rho} C_{k}=\sum_{h=0}^{\varepsilon_{C}} b_{k h} \hat{\boldsymbol{z}}_{C}\left(\mu_{h}\right)=\sum_{h=0}^{\varepsilon_{C}} p_{k}\left(\mu_{h}\right) \hat{\boldsymbol{z}}_{C}\left(\mu_{h}\right)=p_{k} \sum_{h=0}^{\varepsilon_{C}} \hat{\boldsymbol{z}}_{C}\left(\mu_{h}\right)=p_{k} \boldsymbol{\rho} C_{0},
$$

and $C$ is a completely pseudo-regular code.

Consider the vector space $\mathcal{V}_{C}:=\{q \boldsymbol{\rho} C: \forall q \in \mathbb{R}[x]\}$. Since $\left\{Z_{k}^{C}\right\}_{0 \leqslant k \leqslant d_{C}}$ is a basis of $\mathbb{R}_{d_{C}}[x], \mathcal{V}_{C}=\operatorname{span}\left\{\hat{\boldsymbol{z}}_{C}\left(\mu_{0}\right), \hat{\boldsymbol{z}}_{C}\left(\mu_{1}\right), \ldots, \hat{\boldsymbol{z}}_{C}\left(\mu_{d_{C}}\right)\right\}$. Taking in mind that $d_{C} \geqslant \varepsilon_{C}$, the next corollary is obtained.

Corollary 4.5 $C$ is a completely pseudo-regular code if and only if

$$
q \boldsymbol{\rho} C \in \operatorname{span}\left\{\boldsymbol{\rho} C_{0}, \boldsymbol{\rho} C_{1}, \ldots, \boldsymbol{\rho} C_{\varepsilon_{C}}\right\} \quad \text { for all } q \in \mathbb{R}[x],
$$

or, equivalently, if and only if there exists a basis $\mathcal{B}$ of $\mathbb{R}_{d_{C}}[x]$ such that

$$
b \boldsymbol{\rho} C \in \operatorname{span}\left\{\boldsymbol{\rho} C_{0}, \boldsymbol{\rho} C_{1}, \ldots, \boldsymbol{\rho} C_{\varepsilon_{C}}\right\} \quad \text { for all } b \in \mathcal{B} .
$$

An interesting application of this corollary is the following characterization of a completely regular code (for other characterizations, see e.g. [6, 9]).

Theorem 4.6 Let $\Gamma=(V, E)$ be a regular graph. Then $C \subset V$ is a completely regular code if and only if, for any given nonnegative integers $\ell \leqslant d_{C}$ and $k \leqslant \varepsilon_{C}$, the number of $\ell$-walks between (the vertices of ) $C$ and $i \in C_{k}$ does not depend on the vertex $i$.

Proof. In Corollary 4.5 take the canonical basis $\mathcal{B}=\left\{1, x, x^{2}, \ldots, x^{d_{C}}\right\}$ of $\mathbb{R}_{d_{C}}[x]$. Then, there exist constants $\alpha_{h}, 0 \leqslant h \leqslant \varepsilon_{C}$, such that $x^{\ell} \boldsymbol{\rho} C=\sum_{h=0}^{\varepsilon_{C}} \alpha_{h} \rho C_{h}$. Hence,

$$
\begin{aligned}
\left(x^{\ell} \boldsymbol{\rho} C\right)_{i} & =\left(\boldsymbol{A}^{\ell} \sum_{j \in C} \boldsymbol{e}_{j}\right)_{i}=\sum_{j \in C}\left(\boldsymbol{A}^{\ell}\right)_{j i} \\
& =\left(\sum_{h=0}^{\varepsilon_{C}} \alpha_{h} \boldsymbol{\rho} C_{h}\right)_{i}=\sum_{h=0}^{\varepsilon_{C}} \alpha_{h}\left(\sum_{j \in C_{h}} \boldsymbol{e}_{j}\right)_{i}=\sum_{h=0}^{\varepsilon_{C}} \alpha_{h} \delta_{h k}=\alpha_{k} .
\end{aligned}
$$


From this, we get the result.

As the authors of [5] established in the study of the $C$-local pseudo-distance regularity from a combinatorial point of view, the conditions of Theorem 4.2 can be apparently relaxed by restricting them to the set of vertices at maximum distance from $C$, provided that $C$ is extremal. Moreover, this gives a numerical (instead of vectorial) characterization of pseudo-distance-regularity.

Theorem 4.7 Let $\Gamma=(V, E)$ be a graph and let $C \subset V$ be an extremal set with $C$ local spectrum $\operatorname{sp}_{C} \Gamma=\left\{\mu_{0}^{m_{C}\left(\mu_{0}\right)}, \mu_{1}^{m_{C}\left(\mu_{1}\right)}, \ldots, \mu_{d_{C}}^{m_{C}\left(\mu_{d_{C}}\right)}\right\}$. Let $C_{0}, C_{1}, \ldots, C_{\varepsilon_{C}}=\bar{C}$ be the distance partition of $V$ given by the distance to $C$. Then, $C$ is a completely pseudo-regular code if and only if any of the three following conditions applies:

(a) There exists a polynomial $p \in \mathbb{R}_{\varepsilon_{C}}[x]$ such that

$$
p \rho C=\rho \bar{C},
$$

in which case $p=p_{d_{C}}^{C}$.

(b) The highest degree $C$-local predistance polynomial satisfies

$$
p_{d_{C}}^{C}\left(\mu_{0}\right)=\frac{\|\rho \bar{C}\|^{2}}{\|\rho C\|^{2}}
$$

(c) The square norm of the vector $\rho \bar{C}$ is:

$$
\|\boldsymbol{\rho} \bar{C}\|^{2}=\|\boldsymbol{\nu}\|^{2}\left(\sum_{l=0}^{\varepsilon_{C}} \frac{m_{C}\left(\mu_{0}\right) \pi_{0}^{2}(C)}{m_{C}\left(\mu_{l}\right) \pi_{l}^{2}(C)}\right)^{-1} .
$$

Proof. Let us first show that the three conditions are equivalent. To simplify notation, let $p_{k}=p_{k}^{C}$ for $0 \leqslant k \leqslant d_{C}\left(=\varepsilon_{C}\right)$. Then, using Cauchy-Schwarz inequality, we have:

$$
\begin{aligned}
\left\langle p_{k} \boldsymbol{\rho} C, \boldsymbol{\rho} C_{k}\right\rangle & =\left\langle p_{k} \boldsymbol{\rho} C, \boldsymbol{\nu}\right\rangle=\left\langle\boldsymbol{\rho} C, p_{k} \boldsymbol{\nu}\right\rangle=p_{k}\left(\mu_{0}\right)\langle\boldsymbol{\rho} C, \boldsymbol{\nu}\rangle=p_{k}\left(\mu_{0}\right)\|\boldsymbol{\rho} C\|^{2} \\
& \leqslant\left\|p_{k} \boldsymbol{\rho} C\right\|\left\|\boldsymbol{\rho} C_{k}\right\|=\left\|p_{k}\right\|_{C}\|\boldsymbol{\rho} C\|\left\|\boldsymbol{\rho} C_{k}\right\|=\sqrt{p_{k}\left(\mu_{0}\right)}\|\boldsymbol{\rho} C\|\left\|\boldsymbol{\rho} C_{k}\right\| .
\end{aligned}
$$

Hence,

$$
p_{k}\left(\mu_{0}\right) \leqslant \frac{\left\|\rho C_{k}\right\|^{2}}{\|\rho C\|^{2}} \quad\left(0 \leqslant k \leqslant d_{C}\right),
$$

and equality holds if and only if the vectors $p_{k} \rho C$ and $\rho C_{k}$ are colinear. In particular, for $k=d_{C}$, we have that (12) holds if and only if (11) holds with $p=\alpha p_{d_{C}}$ and some $\alpha \in \mathbb{R}$. But, using the same reasonings as in the proof of Corollary 4.3 , we get $\left\langle p, p_{k}^{C}\right\rangle_{C}=0$ for every $k<d_{C}$, and $\|p\|_{C}^{2}=p\left(\mu_{0}\right)$. Consequently, $\alpha=1$ and $p$ is the highest degree $C$-local predistance polynomial, $p=p_{d_{C}}$. This proves the equivalence between $(a)$ and $(b)$.

Let $D=\bar{C}$ be the subset of vertices at distance $\varepsilon_{C}=d_{C}$ from $C$ and assume that $\boldsymbol{\rho} D=p \boldsymbol{\rho} C$. Since $\partial(i, j) \geqslant \varepsilon_{C}$ for every $i \in C$ and $j \in D$, we have $\varepsilon_{D} \geqslant \varepsilon_{C}$. Moreover, 
the equality $p \boldsymbol{\rho} C=p\left(\mu_{0}\right) \hat{\boldsymbol{z}}_{C}\left(\mu_{0}\right)+\cdots+p\left(\mu_{d_{C}}\right) \hat{\boldsymbol{z}}_{C}\left(\mu_{d_{C}}\right)=\boldsymbol{\rho} D$ gives $d_{C} \geqslant d_{D}$. Altogether, we have $\varepsilon_{D} \geqslant \varepsilon_{C}=d_{C} \geqslant d_{D} \geqslant \varepsilon_{D}$, thus $(\varepsilon:=) \varepsilon_{D}=\varepsilon_{C}$ and $(\mathcal{M}:=) \operatorname{ev}_{C} \Gamma=\operatorname{ev}_{D} \Gamma$. This, together with Corollary 2.3(b), proves the equivalence between $(a)$ - $(b)$ and $(c)$ since $\sum_{l=0}^{\varepsilon} m_{D}\left(\mu_{l}\right)=1$.

Now, let us prove that $C$ is a completely pseudo-regular code if and only if any of the conditions holds. The necessity follows from Theorem 3.4 (see also Theorem 4.2). To prove sufficiency, we first note that the orthogonal systems corresponding to the $C$-local predistance polynomials, $\left\{p_{k}\right\}_{0 \leqslant k \leqslant \varepsilon}$, and $D$-local predistance polynomials, $\left\{\bar{p}_{k}\right\}_{0 \leqslant k \leqslant \varepsilon}$, are related in $\mathbb{R}[x] /(Z)$ by $\bar{p}_{k}=p_{\varepsilon}^{-1} p_{\varepsilon-k}, 0 \leqslant k \leqslant \varepsilon$. (This is well-defined since, by Corollary 2.3(a), $p$ has an inverse $p^{-1}$ in the ring $\mathbb{R}_{\varepsilon}[x] /(Z)$, being $Z:=\prod_{l=0}^{\varepsilon}\left(x-\mu_{l}\right)$; see also [2].) Indeed, since $\boldsymbol{E}_{l} \boldsymbol{\rho} D=\boldsymbol{E}_{l} p \boldsymbol{\rho} C=p\left(\mu_{l}\right) \boldsymbol{E}_{l} \boldsymbol{\rho} C$, we have

$$
m_{D}\left(\mu_{l}\right)=\frac{\left\|\boldsymbol{E}_{l} \boldsymbol{\rho} D\right\|^{2}}{\|\boldsymbol{\rho} D\|^{2}}=p^{2}\left(\mu_{l}\right) \frac{\left\|\boldsymbol{E}_{l} \boldsymbol{\rho} C\right\|^{2}}{\|\boldsymbol{\rho} C\|^{2}} \frac{\|\boldsymbol{\rho} C\|^{2}}{\|\boldsymbol{\rho} D\|^{2}}=\frac{p^{2}\left(\mu_{l}\right)}{p\left(\mu_{0}\right)} m_{C}\left(\mu_{l}\right) .
$$

Hence,

$$
\begin{aligned}
\left\langle\bar{p}_{k}, \bar{p}_{h}\right\rangle_{D} & =\sum_{l=0}^{\varepsilon} m_{D}\left(\mu_{l}\right) \bar{p}_{k}\left(\mu_{l}\right) \bar{p}_{h}\left(\mu_{l}\right) \\
& =\frac{1}{p\left(\mu_{0}\right)} \sum_{l=0}^{\varepsilon} m_{C}\left(\mu_{l}\right) p^{2}\left(\mu_{l}\right) p^{-1}\left(\mu_{l}\right) p_{\varepsilon-k}\left(\mu_{l}\right) p^{-1}\left(\mu_{l}\right) p_{\varepsilon-h}\left(\mu_{l}\right) \\
& =\frac{1}{p\left(\mu_{0}\right)} \sum_{l=0}^{\varepsilon} m_{C}\left(\mu_{l}\right) p_{\varepsilon-k}\left(\mu_{l}\right) p_{\varepsilon-h}\left(\mu_{l}\right)=\frac{1}{p\left(\mu_{0}\right)}\left\langle p_{\varepsilon-k}, p_{\varepsilon-h}\right\rangle_{C} \\
& =\delta_{k h} p^{-1}\left(\mu_{0}\right) p_{\varepsilon-k}\left(\mu_{0}\right)=\delta_{k h} \bar{p}_{k}\left(\mu_{0}\right) .
\end{aligned}
$$

Given $0 \leqslant k \leqslant \varepsilon$, let us consider the set $S_{k}=\left\{r+p s: r \in \mathbb{R}_{k-1}[x], s \in \mathbb{R}_{\varepsilon-k-1}[x]\right\}$ where, by convention, $\mathbb{R}_{-1}[x]=\emptyset$. Then, for any $q \in S_{k}$, we have:

$$
\left\langle q \boldsymbol{\rho} C, \boldsymbol{\rho} C_{k}\right\rangle=\left\langle r \boldsymbol{\rho} C, \boldsymbol{\rho} C_{k}\right\rangle+\left\langle s \boldsymbol{\rho} D, \boldsymbol{\rho} C_{k}\right\rangle=0 .
$$

Note also that

$$
\begin{aligned}
\mathbb{R}_{\varepsilon}[x] & =\operatorname{span}\left\{p_{0}, \ldots, p_{k}, p_{k+1}, \ldots, p_{\varepsilon}\right\}=\operatorname{span}\left\{p_{0}, \ldots, p_{k}, p_{\varepsilon} \bar{p}_{\varepsilon-k-1}, \ldots, p_{\varepsilon} \bar{p}_{0}\right\} \\
& =S_{k} \oplus \operatorname{span}\left\{p_{k}\right\}
\end{aligned}
$$

Moreover, using (17), we get that the $C$-local Hoffman polynomial can be written as $H_{C}=q_{k}+\xi_{k} p_{k}$, where $q_{k} \in S_{k}$ and $\xi_{k}$ is the corresponding Fourier coefficient. That is,

$$
H_{C}=q_{k}+\frac{\left\langle H_{C}, p_{k}\right\rangle_{C}}{\left\|p_{k}\right\|_{C}^{2}} p_{k}=q_{k}+m_{C}\left(\mu_{0}\right) H\left(\mu_{0}\right) p_{k}=q_{k}+p_{k} .
$$

Then, from (18), (16) and (14), we get:

$$
\begin{aligned}
\left\langle p_{k} \boldsymbol{\rho} C, \boldsymbol{\rho} C_{k}\right\rangle & =\left\langle\left(H_{C}-q_{k}\right) \boldsymbol{\rho} C, \boldsymbol{\rho} C_{k}\right\rangle=\left\langle H_{C} \boldsymbol{\rho} C, \boldsymbol{\rho} C_{k}\right\rangle=\left\langle\boldsymbol{\nu}, \boldsymbol{\rho} C_{k}\right\rangle=\left\|\boldsymbol{\rho} C_{k}\right\|^{2} \\
& \leqslant \sqrt{p_{k}\left(\mu_{0}\right)}\|\boldsymbol{\rho} C\|\left\|\boldsymbol{\rho} C_{k}\right\| .
\end{aligned}
$$


Thus,

$$
p_{k}\left(\mu_{0}\right) \geqslant \frac{\left\|\rho C_{k}\right\|^{2}}{\|\rho C\|^{2}} \quad\left(0 \leqslant k \leqslant d_{C}\right),
$$

which, together with (15), allows us to conclude that we have equalities in (14), (19), the vectors $p_{k} \boldsymbol{\rho} C, \boldsymbol{\rho} C_{k}$ are colinear for every $0 \leqslant k \leqslant d_{C}\left(=\varepsilon_{C}\right)$, and $C$ is a completely pseudo-regular code by Theorem 4.2.

\section{References}

[1] N. Biggs, Algebraic Graph Theory, Cambridge University Press, Cambridge, 1974; second edition, 1993.

[2] M. Cámara, J. Fàbrega, M.A. Fiol, and E. Garriga, Some families of orthogonal polynomials of a discrete variable and their applications to graphs and codes, Electron. J. Combin 16(1) (2009), \#R83.

[3] M.A. Fiol, On pseudo-distance-regularity, Linear Algebra Appl. 323 (2001), 145-165.

[4] M.A. Fiol and E. Garriga, From local adjacency polynomials to locally pseudodistance-regular graphs, J. Combin. Theory Ser. B. 71 (1997), no. 2, 162-183.

[5] M.A. Fiol and E. Garriga, On the algebraic theory of pseudo-distance-regularity around a set, Linear Algebra Appl. 298 (1999), 115-141.

[6] M.A. Fiol and E. Garriga, An algebraic characterization of completely regular codes in distance-regular graphs, SIAM J. Discrete Math. 15 (2001), 1-13.

[7] M.A. Fiol, E. Garriga, and J.L.A. Yebra, Locally pseudo-distance-regular graphs, J. Combin. Theory Ser. B. 68 (1996), 179-205.

[8] A.J. Hoffman, On the polynomial of a graph, Amer. Math. Monthly 70 (1963), 30-36.

[9] W.J. Martin, Completely regular codes: a viewpoint and some problems, in: Proceedings of 2004 Com2MaC Workshop on Distance-Regular Graphs and Finite Geometry, pp. 43-56, July 24-26, 2004, Pusan, Korea.

[10] H. Suzuki, The Terwilliger algebra associated with a set of vertices in a distanceregular graph, J. Algebraic Combin. 22 (2005), no. 1, 5-38.

[11] P. Terwilliger, The subconstituent algebra of an association scheme (Part I), J. Algebraic Combin. 1 (1992), no. 4, 363-388.

[12] P. Terwilliger, An inequality involving the local eigenvalues of a distance-regular graph, J. Algebraic Combin. 19 (2004), no. 2, 143-172. 